\title{
¿ES COMPATIBLE EL COMPATIBILISMO CON LA EXISTENCIA DE CORRELACIONES CUÁNTICAS DEL TIPO EPR?
}

\author{
JAVIER SÁNCHEZ-CAÑIZARES \\ Universidad de Navarra
}

\begin{abstract}
RESUMEN. El compatibilismo afirma que el libre albedrío es compatible con el determinismo. Para el "compatibilismo de las bifurcaciones» pertenece a la esencia de la libertad «poder actuar de otra manera", mientras que para el "compatibilismo de la fontalidad» resulta decisiva la ultimidad del agente como fuente de control. Ahora bien, dadas dos partículas con entrelazamiento EPR, la medición de una variable en una de ellas determina instantáneamente el valor de dicha variable en la otra. Experimentos recientes hacen finalmente insostenibles explicaciones basadas en variables ocultas locales: 0 la naturaleza está ónticamente indeterminada o bien está determinada de modo global. El primer caso es incompatible con los dos compatibilismos y el segundo es incompatible con el «poder actuar de otra manera». Además, implicaría la existencia de variables ocultas no locales como fuente última de determinación, con longitudes de correlación típicas mayores que las del cuerpo humano: la acción no podría originarse en su interior. El compatibilismo, por tanto, debe ser rechazado.
\end{abstract}

PALABRAS CLAVE: compatibilismo, libertad, mecánica cuántica, correlaciones EPR, problema mente-cerebro.

\section{Is Compatibilism Compatible with the Existence of EPR-like Quantum Correlations?}

ABSTRACT. Compatibilism affirms that free will is compatible with determinism. «Forking path compatibilism» accepts that «being able to act otherwise» belongs to the essence of free will, whereas "sourcehood compatibilism" focus on the ultimacy of the agent as control source. However, in an EPR-entangled pair of particles, measuring of a variable at one of them instantaneously determines the value of the same variable at the other particle. Recent experiments have finally ruled out any explanation of this phenomenon based on local hidden variables, i.e., either nature is ontically undetermined or is globally determined. The former is incompatible with both compatibilisms, while the latter is incompatible with «being able to act otherwise», but also implies acceptance of non-local hidden variables as the ultimate source of determination in nature with correlation lengths typically much bigger than human body's length scales: human action would not ultimately stem from the agent's own interiority. Therefore, compatibilism has to be rejected.

KEY WORDS: compatibilism, free will, quantum mechanics, EPR correlations, mind-brain problem.

\section{El COMPATIBILISMO COMO DOCTRINA FILOSÓFICA}

La doctrina filosófica del "compatibilismo» afirma que el libre albedrío es compatible con el determinismo, es decir, con el hecho de que una vez determinados tanto el estado pasado del universo como las leyes de la naturaleza que lo rigen, únicamente es posible un estado de futuro. Las versiones principales del 
compatibilismo difieren en su modo de concebir la libertad humana: por una parte, el llamado «compatibilismo de las bifurcaciones» (forking path compatibilism) considera que pertenece a la esencia de la libertad "poder actuar de otra manera»; por otra, el denominado "compatibilismo de la fontalidad» (sourcehood compatibilism) se centra en la ultimidad del agente como fuente de control de la acción, que podría resultar a la vez libre y estar determinada por causas interiores al agente. Mientras que el compatibilismo de las bifurcaciones, a pesar de sus intentos de renovación mediante las aportaciones del condicionalismo o del disposicionalismo, parece hoy en día batirse en retirada, el compatibilismo de la fontalidad mantiene una cierta preponderancia gracias a las contribuciones de Harry Frankfurt o John Martin Fischer entre otros.

Aunque los compatibilistas - y los defensores del determinismo en generalson conscientes del cambio de paradigma que se produjo en la física del siglo XX con la aparición de la mecánica cuántica, han solido considerar el indeterminismo cuántico como una manifestación secundaria - probablemente a causa de la imperfección de nuestro conocimiento- de un determinismo fundamental de la naturaleza. A favor de dicha postura pueden aducirse una serie de interpretaciones no estándar de la mecánica cuántica que presentan un indeterminismo mitigado o simplemente lo niegan. Sin embargo, se ha prestado poca atención hasta ahora a la compatibilidad de las diversas versiones del compatibilismo con la existencia, cada vez mejor probada, de correlaciones cuánticas de tipo EPR en la naturaleza.

\section{El EXPERIMENTO MENTAL EPR}

En un famoso artículo de 1935, Albert Einstein, Boris Podolsky y Nathan Rosen (EPR) idearon un experimento mental que pretendía demostrar la insuficiencia de la mecánica cuántica como teoría física fundamental. El experimento puede describirse en pocas líneas. Supongamos un sistema en reposo que se desintegra en dos partículas similares. Debido a los principios de conservación del momento lineal y angular, ambas partículas viajan en sentidos opuestos con la misma velocidad y, además, si se mide el espín (momento angular interno) de una de ellas, instantáneamente sabremos cuál es el espín de la otra —será justo su opuesto, al estar totalmente anticorrelacionado con el de su pareja - sin necesidad de haber realizado ninguna medida sobre esta última. Puesto que, según la teoría de la relatividad, no puede darse una interacción física que viaje a velocidad mayor que la de la luz, para EPR sería absurdo pensar que la medición del espín de una partícula pudiera estar influyendo en el espín de la otra, que habría de estar determinado (aunque nosotros desconozcamos su valor) antes de que se realizara la medición. Así pues, el indeterminismo profesado por la mecánica cuántica — que en su interpretación estándar considera que las magnitudes físicas no están determinadas antes de que se haga una medición de las mismas- no sería fundamental, sino que reflejaría meramente nuestra ignorancia de las variables locales (ocultas, pues no las conocemos) que determinan de antemano el valor del espín de cada partícula. 


\section{Las desigualdades de Bell}

Pudiera parecer que estamos ante una cuestión metafísica o de interpretación de una teoría, pues no cambiaría los resultados el que la indeterminación fuese real o que hubiese una determinación inaccesible a nuestro conocimiento. Pero no es así. En los años 60, gracias a los trabajos del físico irlandés John Bell, se encontró la manera de discriminar experimentalmente entre una naturaleza gobernada por variables ocultas locales - tal y como defendía el artículo EPR - y una naturaleza indeterminista, aleatoria y no-local — de acuerdo con la interpretación estándar de la mecánica cuántica. Bell demostró que la existencia de variables ocultas locales que determinan totalmente el estado de un sistema implicaría la presencia de un límite en las correlaciones que puede haber entre los valores estadísticos de las medidas que se hacen en el sistema. Dicho límite se expresa mediante las llamadas desigualdades de Bell, que han de satisfacer los sistemas clásicos deterministas. Por el contrario, en el caso de que la interpretación de la mecánica cuántica fuese correcta, podrían existir correlaciones inalcanzables dentro de la perspectiva de la física clásica, aun con variables ocultas. La mecánica cuántica, en definitiva, predice la violación de las desigualdades de Bell en determinadas situaciones.

\section{LOS EXPERIMENTOS REALES EPR}

En el campo experimental, se han producido hasta la fecha diversos intentos de realizar este test. Probablemente, el más conocido durante el siglo XX fue llevado a cabo por Alain Aspect y su equipo en el Instituto de Óptica de Palaiseau (Francia). Si bien las medidas de los espines o la polarización de las partículas elegidas (que sirve también para nuestros propósitos) resulta cada vez menos problemática en el plano técnico, los resultados obtenidos hasta 2015 no gozaban de consenso generalizado en la comunidad científica a causa de dos «agujeros» conceptuales importantes en la realización del experimento: (1) Si las partículas elegidas eran fotones, se presentaba el llamado «problema de la detección». Los fotones son difíciles de controlar en su totalidad y cabía la posibilidad de que se estuviese realizando la detección de una muestra no representativa, que conllevara algún sesgo incontrolado en los resultados. (2) Si las partículas utilizadas eran electrones, más lentos y más fáciles de controlar, surge el "problema de la comunicación». No sería del todo descartable que los electrones que formasen la pareja EPR estuviesen lo suficientemente cerca para poder comunicarse (obviamente a velocidad menor que la de la luz) sin que llegáramos a saberlo, invalidando así los presupuestos teóricos del experimento.

Sin embargo, en agosto de 2015, el equipo liderado por Ronald Hanson en la Universidad Tecnológica de Delft ha sido capaz por primera vez de llevar a cabo un test EPR libre de los dos problemas antes mencionados. En el experimento, se combinan electrones —alejados entre sí una distancia de 1,3 kilómetros- con fotones, que entran en comunicación entre sí en un tercer lugar para lograr formar un estado cuántico entrelazado de las cuatro partículas. Aunque la situación es conceptualmente más compleja que la presentada inicialmente en el experimento 
mental EPR, las desigualdades de Bell correspondientes a la nueva situación mantienen su capacidad de discriminar entre las correlaciones clásicas y cuánticas. El resultado del experimento no arroja lugar a dudas: a partir de 245 ensayos, el valor promedio de las correlaciones halladas entre las diversas medidas ha sido de $2,42 \pm 0,20$, mientras que las correlaciones clásicas habrían de ser siempre menores que 2. Lo que había sido incoado en experimentos anteriores, resulta confirmado con la mayor fuerza posible con estos nuevos resultados.

\section{Conclusiones}

¿Qué posibilidades quedan entonces para la interpretación de la realidad a partir de la constatación de la existencia de correlaciones cuánticas tipo EPR? O bien, como defiende la interpretación estándar de la mecánica cuántica, la naturaleza está ónticamente indeterminada o bien está globalmente determinada, de modo no local, pues los experimentos rechazan la existencia de variables ocultas locales, pero no la posibilidad de un superdeterminismo en el universo. Pero si estas son las dos únicas posibilidades, ninguna de las dos versiones del compatibilismo que hemos mencionado al principio de esta contribución puede mantenerse: obviamente, la indeterminación óntica de la naturaleza es incompatible con cualquier doctrina compatibilista y la superdeterminación global es incompatible con «poder actuar de otra manera» y, por tanto, con el compatibilismo de las bifurcaciones. Pero dicho superdeterminismo, además, implicaría la existencia de variables ocultas no locales como fuente última de determinación para la naturaleza, con longitudes de correlación espacio-temporales típicamente mayores que las del cuerpo humano, de modo que la acción no podría originarse en último término a partir de la interioridad del agente, como sostiene el compatibilismo de la fontalidad. La conclusión necesaria es que la existencia de correlaciones cuánticas del tipo EPR, probada fehacientemente en agosto de 2015, excluye ambas versiones del compatibilismo.

Grupo «Mente-cerebro» del Instituto Cultura y Sociedad (ICS) y

JaVIER SÁNCHEZ-CAÑIZARES

Grupo de Investigación «Ciencia, Razón y Fe» (CRYF)

Universidad de Navarra

js.canizares@unav.es

[Artículo aprobado para publicación en diciembre de 2016] 